\title{
Propuesta de unidad didáctica de lucha olímpica: Luchas del Mundo
}

\author{
Alberto MARTÍNEZ-ABELLÁN* \\ Universidad de Murcia (España)
}

I Congreso Nacional de Entrenadores de Judo, Murcia (España), 13-15 de julio, 2018

\section{Resumen}

La lucha es una actividad natural del ser humano y está representada en una gran cantidad de países y culturas. En la educación secundaria los deportes de lucha pueden ayudar a los estudiantes a desarrollar destrezas y actitudes importantes. Con los ejercicios de lucha se desarrollan capacidades motrices, perceptivas y afectivas, ya que se trata de un deporte de contacto. El objetivo de este trabajo fue diseñar una unidad didáctica bilingüe dirigida a la etapa de secundaria, donde a través de ejercicios de lucha se puedan alcanzar objetivos didácticos relacionados con la lucha olímpica y fomentar situaciones de comunicación en inglés. La unidad didáctica, llamada Luchas del Mundo / World Wrestling, tendrá un carácter lúdico e integrador, permitiendo relaciones y comunicaciones entre los compañeros/as en las clases prácticas con un leguaje en inglés. Esta propuesta consta de seis sesiones, realizando un recorrido por diferentes continentes y llevando a cabo ejercicios específicos de lucha, para finalizar con las modalidades olímpicas de lucha. Se llevará a cabo un diseño similar a lo largo de todas las prácticas, realizando un calentamiento general y específico; una parte principal, en la que se ejecutarán los ejercicios luctatorios más representativos, como agarres, desequilibrios, inmovilizaciones, etc.; y una vuelta a la calma con estiramientos y conclusiones. En definitiva, se pretende continuar con los contenidos trabajados en primaria y extender la lucha olímpica en secundaria.

Palabras clave: Lucha olímpica; deportes de combate; unidad didáctica; educación secundaria; inglés.
\end{abstract}

\begin{abstract}
Wrestling is a natural human activity and it is represented in a large number of countries and cultures. In secondary education, combat sports can help students develop relevant skills and attitudes. Wrestling exercises develop motor, perceptual and affective capacities, since wrestling is a close contact sport. The objective of this work was to design a bilingual teaching unit aimed at the secondary education, where wrestling exercises are used to achieve educational purposes related to learning Olympic Wrestling and fostering situations of communication in English. The didactic unit, called Luchas del Mundo / World Wrestling, will have a recreational and integrative character, and will allow students to relate and communicate with their peers in English during the practical sessions. The didactic unit consists of six sessions, making a journey through different continents and wrestling styles, and finishing with the Olympic wrestling styles. All sessions will follow a similar scheme, starting with a general and specific warm up; following with the main part, in which representative wrestling exercises will be practiced (grips, holds, unbalancing, etc.); and finishing with a cool down, stretches and conclusions. In summary, this proposal follows the contents worked in primary education physical education and tries to promote the practice of Olympic Wrestling in secondary education.
\end{abstract}

Keywords: Olympic Wrestling; combat sports; didactic unit; secondary education; English.

\section{Contextualización}

La lucha ha estado presente en todas las culturas y es una actividad natural del ser humano. Tiene un núcleo central que es la relación con otra persona, pero además es un deporte de tradición, rituales, utilidad, actividad física, etc. (Atencia, 2000). Existe una implicación dual constante, mediante la cual se desarrolla una noción oponente-colaborador que es muy importante en los objetivos didácticos (Avelar \& Figueiredo, 2009). La gran variedad de actividades, juegos y ejercicios para impartir las clases de educación física es una ventaja beneficiosa para los alumnos/as, que desarrollaran un amplio abanico de posibilidades de educación dentro de la etapa de secundaria (Blázquez, 1995; Consejo Superior de Deportes, 2010). Basándonos en el Decreto no 220/2015, de 2 de septiembre de 2015, por el que se establece el currículo básico de la Educación

\footnotetext{
*Email: alberto.martinez1@um.es
} 
Secundaria Obligatoria en la Comunidad Autónoma de la Región de Murcia, los deportes de lucha tienen una gran cabida, ya que mediante su práctica los escolares podrán adquirir actitudes y destrezas necesarias para su desarrollo personal. Se adquieren connotaciones educativas en función de los requerimientos energéticos, perceptivos y afectivos, ya que se establece una relación muy próxima e íntima con los compañeros/as, al realizar ejercicios de intentar derribar o fijar al suelo al adversario (Santos \& Prieto 2010). Se mantiene un contacto físico durante todo el combate o tarea.

Teniendo en cuenta todo lo anterior y nuestra experiencia profesional, hemos diseñado una unidad didáctica bilingüe dirigida a la etapa de secundaria donde, a través de las luchas del mundo, se podrán alcanzar los objetivos didácticos y fomentar las situaciones de comunicación en inglés (Rodríguez, 2009). La unidad didáctica, llamada Luchas del Mundo / World Wrestling, tendrá un carácter lúdico e integrador, permitiendo relaciones y comunicaciones entre los compañeros/as en las clases prácticas con un leguaje en inglés. Esta propuesta didáctica se basa en promover la comunicación inglesa en diferentes contextos. Para ello se utilizará un vocabulario estándar y uno más específico para el desarrollo de la unidad didáctica. Todo esto ayudará a los alumnos/as a adquirir una confianza en su expresión oral, para posteriormente aplicar sus conocimientos en sus relaciones sociales, académicas o laborales de carácter internacional (Rodríguez, 2009). Existe una gran relación interdisciplinar entre la asignatura de Inglés y Educación Física, por lo que el profesor de inglés tiene que ayudar a aumentar el vocabulario más específico y el profesor de educación física debe tener un buen nivel de inglés para llevar a cabo correctamente todas las sesiones.

\section{Objetivos}

Dentro de esta etapa educativa los objetivos didácticos están sujetos a diversos factores, como son las demandas del currículo, la intención docente, las características de los alumnos/as y el contexto. Para esta unidad didáctica destacaríamos:

- Recopilar un vocabulario específico de lucha en inglés y ponerlo en práctica durante las sesiones.

- Favorecer el contacto físico y motivar hacia la práctica deportiva de actividades luctatorias.

- Reconocer el enfrentamiento cuerpo a cuerpo con una oposición real y directa.

- Experimentar las distintas formas de desequilibrar, derribar, inmovilizar, caer y voltear.

- Realizar ejercicios con elementos técnicos, tácticos, estratégicos y reglamentarios de lucha.

- Adquirir nociones con distintos roles (atacante, defensor, árbitro, espectador, etc.).

- Aceptar la práctica de lucha con deportividad y respeto y no con agresividad.

- Sensibilizar sobre la importancia de la colaboración, con actividades cooperativas y haciendo ver la importancia del adversario como pareja indispensable.

\section{Propuesta}

Las seis sesiones que se llevarán a cabo en esta unidad didáctica serán un supuesto recorrido por las luchas del mundo, lo que nos facilitará una buena introducción en las modalidades de luchas olímpicas. En cada clase se realizarán unos ejercicios de lucha específicos (Tabla 1) y serán asignados a un continente con juegos de algunos países, como por ejemplo los elefantes indios, peleas de monos, lucha brasileña, etc. En todas las sesiones se usará el vocabulario específico en inglés. En el trascurso de las seis sesiones se desarrollará un esquema muy similar, en el cual, se hará una introducción al principio de cada clase práctica explicando el tipo de lucha que se desarrolla en cada continente. A continuación, se realizará un calentamiento, que en las primeras sesiones será con un carácter más lúdico, para pasar después a introducir un calentamiento más específico. En la parte principal se desarrollarán los ejercicios y juegos principales de cada sesión. Por último, en la vuelta a la calma, se realizarán ejercicios de estiramientos y/o relajación, pero 
también se pondrán en común todas las experiencias vividas y posibles mejoras para las siguientes sesiones.

Tabla 1. Principales contenidos de las sesiones

\begin{tabular}{|c|c|}
\hline $\begin{array}{l}\text { SESIÓN 1: La Lucha en Asia. } \\
\text { / Wrestling in Asia }\end{array}$ & $\begin{array}{l}\text { - Introducción histórica de las luchas del mundo. } \\
\text { - Explicación del desarrollo del vocabulario especifico de lucha en inglés. } \\
\text { - Juegos de empujar (empujar la muralla China, pulso iraní, elefante indios, } \\
\text { lucha coreana, sumo). } \\
\text { - Evaluación inicial. }\end{array}$ \\
\hline $\begin{array}{l}\text { SESIÓN 2: La Lucha en } \\
\text { África / Wrestling in Africa }\end{array}$ & $\begin{array}{l}\text { - Desplazamientos y movimientos corporales. } \\
\text { - Ejercicios de caídas voluntarias e involuntarias. } \\
\text { - Juegos de lucha de oposición en grupos (gorila africano, lucha de la selva). } \\
\text { - Ejercicios luctatorios de oposición por el territorio (peleas de monos, lucha } \\
\text { del Congo, lucha senegalesa). }\end{array}$ \\
\hline $\begin{array}{l}\text { SESIÓN 3: La Lucha en } \\
\text { América / Wrestling in } \\
\text { America }\end{array}$ & $\begin{array}{l}\text { - Juegos de lucha de oposición dual. } \\
\text { - Ejercicios de giros e inmovilizaciones (lucha argentina, lucha cubana, lucha } \\
\text { brasileña, lucha de México). } \\
\text { - Actividades de lucha en suelo (lucha canadiense, lucha escolar } \\
\text { estadounidense). }\end{array}$ \\
\hline $\begin{array}{l}\text { SESIÓN 4: La } \\
\text { Europa I / W } \\
\text { Europe I }\end{array}$ & $\begin{array}{l}\text { - Juegos y actividades de lucha. } \\
\text { - Ejercicios de agarre (hilera alemana, círculo de la lucha francesa), } \\
\text { - Tareas de desequilibrios y derribos (lucha de vikingos, lucha celta). }\end{array}$ \\
\hline $\begin{array}{l}\text { SESIÓN 5: La Lucha en } \\
\text { Europa II / Wrestling in } \\
\text { Europe II }\end{array}$ & $\begin{array}{l}\text { - Juegos y actividades de lucha. } \\
\text { - Enfrentamientos duales con agarre y control (espartanos, batalla griega). } \\
\text { - Lucha en pie: desequilibrios y derribos (lucha canaria, lucha turca). }\end{array}$ \\
\hline $\begin{array}{l}\text { SESIÓN 6: Lucha Olímpica / } \\
\text { Olympic Wrestling. }\end{array}$ & $\begin{array}{l}\text { - Diferenciación de las modalidades olímpicas. } \\
\text { - Técnicas básicas para llevar a suelo (pasada atrás, entradas piernas). } \\
\text { - Técnicas básicas para dar la vuelta al compañero/a (doble tracción de } \\
\text { brazos y piernas, palanca). } \\
\text { - Ejercicios de lucha olímpica. }\end{array}$ \\
\hline
\end{tabular}

El desarrollo de la unidad didáctica se basa en ejercicios específicos de lucha (empujón, tracción, derribos, etc.) pero con nombres de países o similares para que sean más atractivos para los alumnos/as, y además realizar un recorrido por otras culturas de todo el mundo. La evaluación se llevará a cabo mediante fichas de observación diarias, para observar la evolución de los alumnos/as durante toda la unidad didáctica.

\section{Conclusiones}

Esta propuesta de unidad didáctica está diseñada para continuar trabajando los contenidos de lucha olímpica que se han adquirido en primaria. La Región de Murcia incluye esta modalidad dentro del programa de Deporte en Edad Escolar con el programa de "Nano-Nana práctica grecorromana", en el que se imparten unidades didácticas en los colegios y se disputa una fase regional. Por lo tanto, a través de esta proposición se puede continuar acercando la lucha olímpica a los alumnos de secundaria y seguir promocionando este deporte. La proposición tiene un carácter lúdico, ya que para practicar el deporte de lucha olímpica se requiere de bastantes conocimientos y de esta forma jugada se puede llegar a todo el alumnado.

\section{Referencias}

Atencia, D. (2000). Deportes de lucha. Barcelona: INDE.

Avelar, B., \& Figueiredo, A. (2009). La iniciación a los deportes de combate: interpretación de la estructura del fenómeno lúdico luctatorio. Revista de Artes Marciales Asiáticas, 4(3), 44-57. doi: $10.18002 /$ rama.v4i3.177

Blázquez, D. (1995). La iniciación deportiva y el deporte escolar. Barcelona: INDE.

Consejo Superior de Deportes. (2010). Proyecto marco nacional de la actividad física y el deporte en edad escolar. Madrid: Consejo Superior de Deportes. 
Decreto no 220/2015, de 2 de septiembre de 2015, por el que se establece el currículo de la Educación Secundaria Obligatoria en la Comunidad Autónoma de la Región de Murcia (BOM no 203, de 3 de septiembre).

Rodríguez, M. (2009). Unidad didáctica bilingüe de pre-lucha: be water my friend!. EmásF. Revista Digital de Educación Física, 1(6). Recuperado de https://dialnet.unirioja.es/descarga/articulo/3324145.pdf

Santos, L., \& Prieto, J.A. (2010). Desarrollo de las capacidades perceptivo-motrices a través de las habilidades básicas de lucha en el área de educación física. Sevilla: Wanceulen. 